\title{
The role of EMODnet Chemistry in the European challenge for Good Environmental Status
}

\author{
Matteo Vinci, Alessandra Giorgetti, and Marina Lipizer \\ OGS (Istituto Nazionale di Oceanografia e di Geofisica Sperimentale) Borgo Grotta Gigante 42/C, \\ 34010, Sgonico (TS), Italy \\ Correspondence to: Matteo Vinci (mvinci@inogs.it)
}

Received: 22 June 2016 - Discussion started: 25 July 2016

Revised: 14 December 2016 - Accepted: 10 January 2017 - Published: 15 February 2017

\begin{abstract}
The European Union set the ambitious objective to reach within 2020 the goal of Good Environmental Status. The European Commission (2008) represents the legislative framework that drives member state efforts to reach it. The Integrated Maritime Policy supported the need to provide a European knowledge base able to drive sustainable development by launching in 2009 a new European Marine Observation and Data Network (EMODnet). Through a stepwise approach, EMODnet Chemistry aims to provide high-quality marine environmental data and related products at the scale of regions and sub-regions defined by the Marine Strategy Framework Directive. The chemistry lot takes advantage and further develops the SeaDataNet panEuropean infrastructure and the distributed approach, linking together a network of more than 100 National Oceanographic Data Centres providing data from more than 500 data originators. The close interaction with EEA, RSCs, ICES and EMODnet-MSFD coordination group facilitated the identification of the most appropriate set of information required for the MSFD process. EMODnet Chemistry provides aggregated and validated regional data collections for nutrients, dissolved gasses, chlorophyll, and contaminants, properly visualized with OGC WMS and WPS viewing services. Concentration maps with 10-year moving window from 1960 to 2014 , by season and for selected vertical layers, are computed and made available.
\end{abstract}

\section{Introduction}

The European Union has set the ambitious objective to reach within 2020 the goal of Good Environmental Status (GES) for our oceans and seas. The challenge consists in facing the environmental degradation caused by years of unsustainable and inefficient growth model. The Marine Strategy Framework Directive (MSFD; European Commission, 2008) adopted in 2008, with its 11 descriptors and related indicators, represents the legislative framework and the backbone of this work. MSFD defines the GES in Article 3 as "The environmental status of marine waters where these provide ecologically diverse and dynamic oceans and seas which are clean, healthy and productive". GES means that the different uses made of the marine resources are conducted at a sustainable level, ensuring their continuity for future generations. Going more in detail for the restoration and the safeguard of this status, the ecosystems (including their hydromorphological, physical, and chemical conditions) should be fully functioning and resilient to human-induced environmental change, the decline of biodiversity caused by human activities should be prevented, and biodiversity should be protected. The human activities (introducing substances and energy) should not cause pollution effects and noise from human activities should be compatible with the marine environment and its ecosystems.

This process is at the moment halfway between the adoption (2008) and its deadline (2020). The main features of this strategy are the ecosystem approach (to provide an integrated evaluation of the activities affecting our seas) and the common efforts required by the member states for the cooperation between neighbouring countries. 


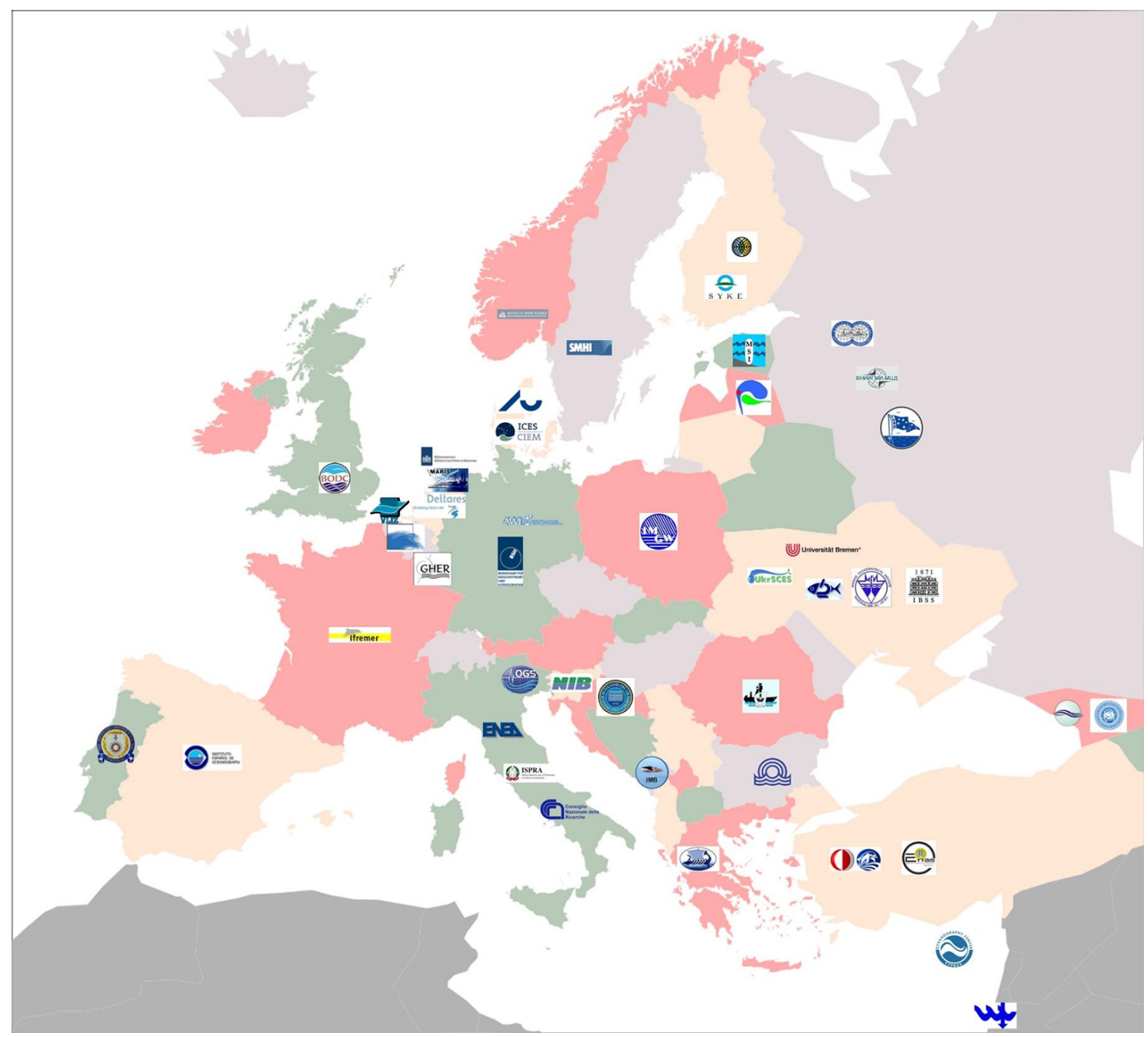

Figure 1. Geographic coverage of EMODnet Chemistry partnership. Logos indicate the nationality of the partner institutes.

Some efforts have already been undertaken by European member states which provided in 2012 the initial assessment on the state of the environment of the national marine waters. This assessment reported on environmental status determined in a holistic way, according to 11 descriptors, and on the objectives and targets to reach GES following the articles 8,9 , and 10 of the MSFD.

The results of the first phase allowed us to recognize gaps and needs in data availability, large heterogeneity of methodological approaches to report information, and spatial inconsistency within member states regarding coastal-offshore data. These outcomes clearly indicated that more efforts are urgently needed if the EU wants to reach its goal. More has to be done in regards to cooperation and especially the integration between member states and regional sea conventions (RSC). The report from the commission on the first phase of implementation of MSFD indicates a high level of heterogeneity among member state reports and in several cases poor data availability and accessibility (Dupont et al., 2014; Palialexis et al., 2014).
As a consequence, evaluation at higher level (regional and EU) is difficult to perform. This first phase of MSFD implementation has somehow brought Europe one step closer to the ecosystem approach. However, the recognized gaps in data and information and the high heterogeneity in assessment approaches should encourage the stakeholders involved in MSFD implementation to develop a more homogeneous approach. In light of the revision of the assessment in 2018, several efforts are required to overcome the shortcomings identified in the first reporting phase. Going more in detail, the actions should be focused on different aspects like revised criteria for GES, methodological standards and standardized methods for monitoring, assessment and data availability, and implementation of integrated information systems at regional and EU level.

In the field of marine research, during the last decades several oceanographic data management initiatives faced the challenge of data availability, interoperability, and resilience at pan-European level (EU MAST MTP II MATER 19961999, EU MAST-INCO MEDAR 1999-2001, FP6 SeaDataNet 2006-2011, FP7 SeaDataNet2 2011-2015). 


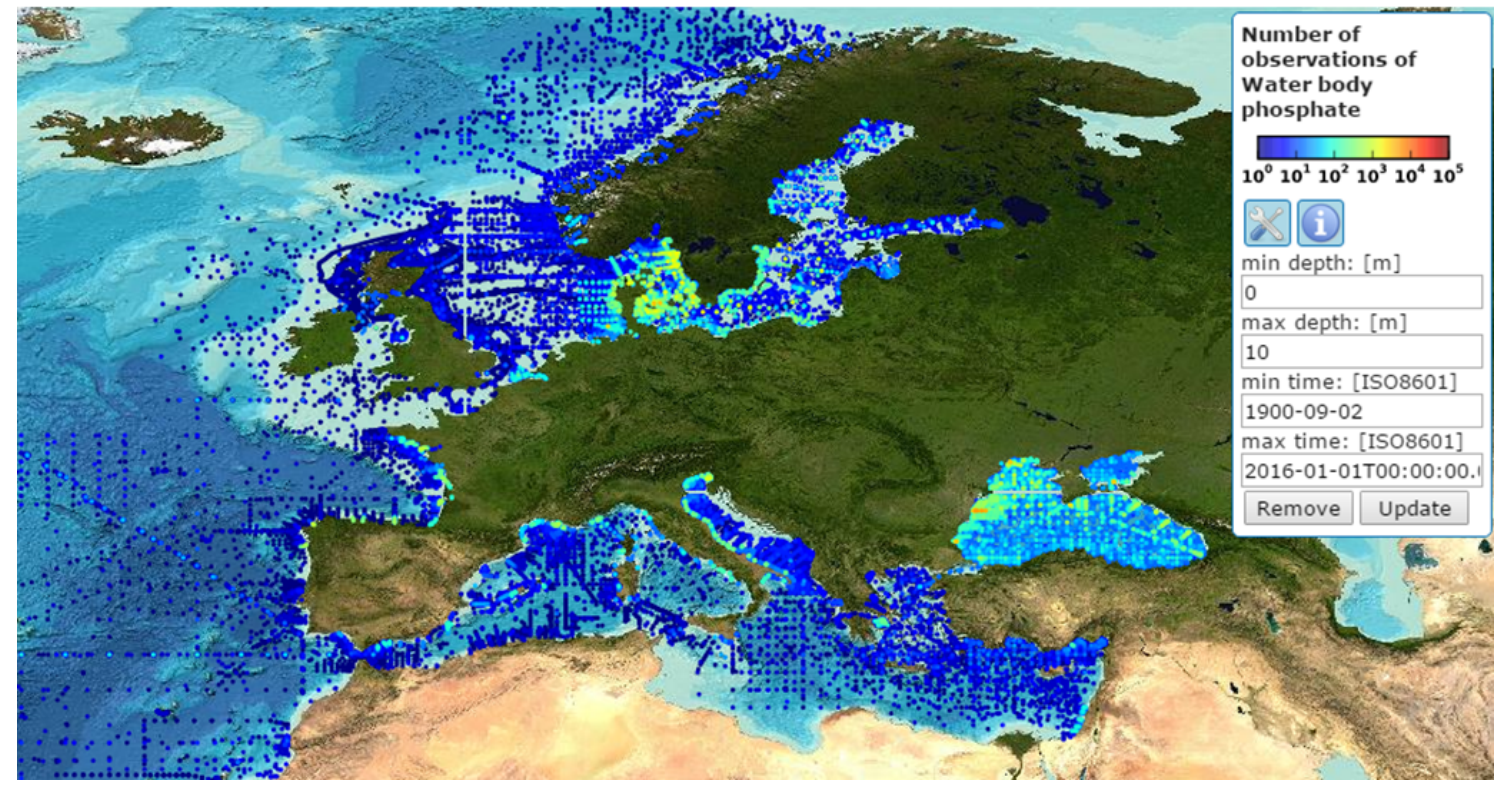

Figure 2. Density and distribution of water body phosphate harvested stations

Interoperability is defined as "the ability of a system to work with or use the parts of another system", while resilience is defined as "the ability of a system to cope with change". The translation of these principles in the oceanographic data management consists in the development of a long life system able to easily interact with other systems. As example the adoption of common formats for data and metadata and a system of common vocabularies ensure that the network of involved persons is working in a homogeneous environment from the syntactic and semantic point of view (speaking a common language). The resilience is safeguarded by metadata and quality flags that provide clear knowledge of which kind of information the users are handling even long time after the data measurement (e.g. use of historical data for time series studies).

Since 2007, the Directive establishing an Infrastructure for Spatial Information in the European Community (INSPIRE, European Commission, 2007) has been the driving principle to ensure that the European spatial data infrastructures are compatible and usable in a transnational context. The directive requires that common implementing rules are adopted for the organization, accessibility, and sharing of spatial information with a focus to the implementation of interoperability of spatial datasets and services. Marine data management communities, developed in the framework of European initiatives such as the abovementioned MATER (1996-1999) and MEDAR (1999-2001) that converged later in the SeaDataNet (2006-2015) experience, faced the challenge to provide access to the huge amount of already existing but fragmented and inaccessible data collected by EU oceanographic institutes. This was done developing a system able to collect, standardize, quality control (QC), and share the information, taking into proper account the data policies.

The simple but efficient idea was the active collection of the EU oceanographic data at national level carried out by a network of National Oceanographic Data Centres (NODCs). The collection of those data was done in direct communication with the data originators to ensure the best set of measured data and related metadata. Metadata, which are all the information needed to describe exhaustively the data, reply to a set of basic but fundamental questions - who, where, when, what, and how - about the collected information. For this reason they are key elements to enable efficient browsing and discovering.

Between the data collection and sharing, the crucial steps to ensure interoperability and reliability consist in standardization and QC.

The standardization is done at two main levels by following the interoperability principles provided by INSPIRE: syntactic and semantic. The first is done providing common formats for the files providing metadata and data (XML ISO, ASCII). The second is done by means of a set of common vocabularies that let to "use the same language" to describe data and metadata over time, different projects, and nationality.

The QC procedures provide the necessary labelling to complete the harvested information with the evaluation of their reliability.

Finally the registered users can access the needed information according to data access and usage policies defined in agreement with the originators.

In order to extend this approach to different disciplines of the marine environment, at EU level, the Directorate-General 


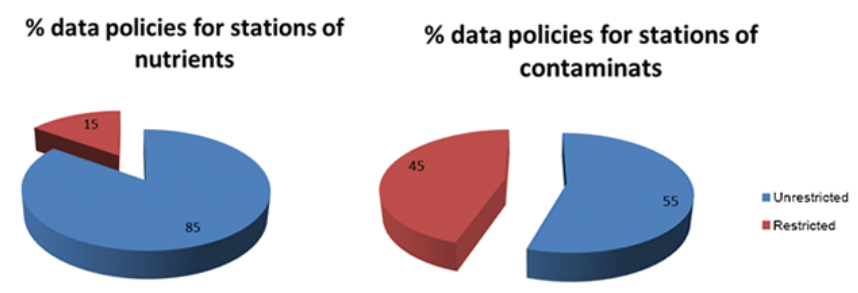

Figure 3. Data policy for nutrients and contaminant data.

for Maritime Affairs and Fisheries (DG-MARE) launched in 2009 a set of thematic contracts to establish a European Marine Observation and Data Network (EMODnet). The aim of the initiative was to improve the availability of highquality marine environmental data at the scale of regions and sub-regions of the Marine Strategy Framework Directive to build a knowledge base that can assist in the implementation of marine policies and drive sustainable development. The EMODnet lots with their infrastructure could play a central role specifically for countries where the RSC are less mature to support the need of qualified and standard information at national, regional, and bigger scales.

\section{Background}

A pilot project was launched by DG-MARE in 2009 to create the components of the European Marine Observation and Data Network (so called ur-EMODnet), as proposed in the EU Green Paper on Future Maritime Policy (European Commission, 2006), consisting of six thematic data portals managing data on bathymetry, marine geology, chemistry, biology, seabed habitats, and physical oceanography. Based on the successful experience of the SeaDataNet project (7th Framework Program), EMODnet Chemistry adopted its approach (Vinci et al., 2013). The principle was to take advantage of its efficient and distributed infrastructure for the management of data deriving from in situ and remote observation of seas and oceans. This infrastructure can be considered a European de facto standard, as it already involves around 100 institutes (nodes) from 35 countries and is adopted and continuously adapted according to specific requirements for chemical data management.

SeaDataNet is actively involved in the development of standards that follow the INSPIRE implementing rules to ensure interoperability such as

- common metadata standards based on the Extensible Mark-up Language (XML), based on ISO 19115/19139 schema;

- standard data transport formats Ocean Data View (ODV) ASCII, MEDATLAS and NetCDF (CF);

- common QC methods and quality flag scale;
- common Vocabulary Web services, used to mark up metadata and data, covering a broad spectrum of disciplines and governed by an international board (SeaVox);

- SOAP Web services for various communication tasks;

- Open Geospatial Consortium (OGC) compliant services (Web Map Service, Web Feature Service, Web Processing Services) for viewing services of data products.

The partnership involved a subgroup of the SeaDataNet network of NODCs with specific experience in data collection, in data analyses, validation, and creation of products and in the technical partners who further developed SeaDataNet infrastructure. The Chemistry Pilot project was focused on the collection and management of data on some chemical parameters relevant for the MSDF (contaminants and fertilizers), in three matrices (sediment, seawater, and biota) and in three areas of interest: the North Sea, the Black Sea, and some spots in the Mediterranean Sea.

The comparison of the harvested data between sea basins highlighted a highly heterogeneous situation according to the different parameters. Data distribution consisted, on one hand, in coastal time series stations monitored at regular temporal scale and, on the other hand, in data homogenously distributed at basins level, but discontinuously in time. Furthermore, high heterogeneity in data managed resulted in the different sampling and analytical protocols adopted, as well as in the different target species. As a last step of the pilot project data visualizations were provided as interpolated maps when data were homogeneously distributed in time and space and as time series plots to allow visualization of data with fragmented spatial coverage. The viewing products were made available on the dedicated web portal in OGC compliant format (WMS layers).

\section{EMODnet}

The positive outcomes from the pilot project confirmed the interest in the further development of a marine observation infrastructure able to provide data and knowledge required to support the development of marine economy whilst supporting environmental protection needs, as underlined in the Green Paper Marine Knowledge 2020 (European Commission, 2012). The new phase includes data collection for all European sea-basins, the Baltic Sea, the northeast Atlantic Ocean, the Mediterranean Sea, and the Black Sea, and involves 46 partners (Fig. 1), from both research institutes and national monitoring agencies.

Data managed by EMODnet now include also silicates, chlorophyll, partial pressures of dissolved gases (oxygen and carbon dioxide), plastics (polyethylene, polypropylene) and acidity ( $\mathrm{pH}, p \mathrm{CO}_{2}$, total inorganic carbon, alkalinity).

Data collection and product generation for all European basins is carried out by five regional leaders, responsible 
Table 1. Number of stations for the parameters with more data available.

\begin{tabular}{ll}
\hline Parameter & No. \\
\hline Phosphate concentration parameters in the water column & 305896 \\
Nitrate concentration parameters in the water column & 262378 \\
Silicate concentration parameters in the water column & 245755 \\
Dissolved oxygen parameters in the water column & 198357 \\
Ammonium and ammonia concentration parameters in water bodies & 188666 \\
Nitrite concentration parameters in the water column & 181642 \\
Salinity of the water column & 151969 \\
Chlorophyll pigment concentrations in water bodies & 145374 \\
\hline
\end{tabular}

for the North Sea, the Baltic Sea, the Atlantic, the Mediterranean, and the Black Sea.

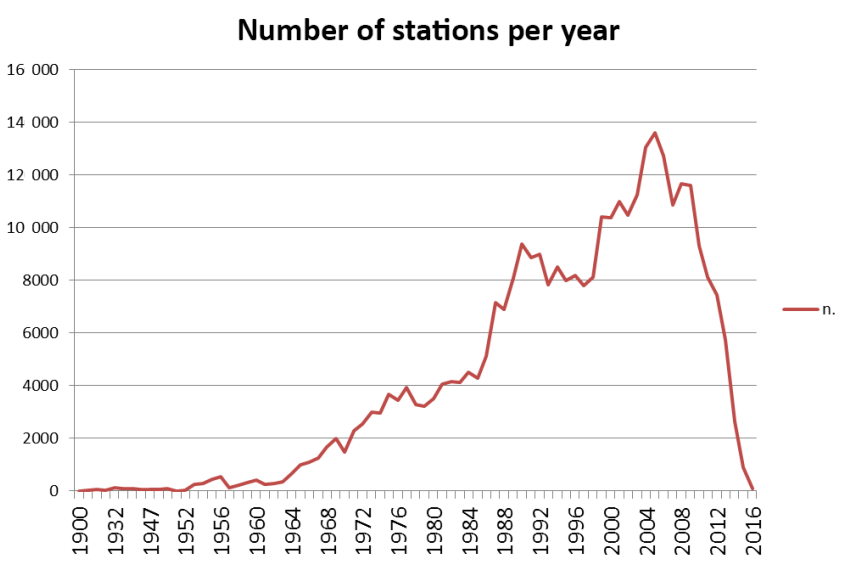

Figure 4. Temporal distribution of nutrient data, spanning from 1900 to 2016 (counting 90 profiles in the current year; updates May 2016).

In order to better tune EMODnet efforts for the requirements of the MSFD, several initiatives have been carried out to strengthen the dialogue with the RSC and the Marine Observation and Data Expert Group (MODEG) and a MSFD-EMODnet coordination group involving RSC, member states, and relevant stakeholders has been established jointly by DG Mare and DG Environment. In addition, regular meetings with INSPIRE implementing groups are organized to discuss on the most feasible and useful products and services to provide.

\section{Data collection and access}

Data harvesting is a fundamental activity of EMODnet and it is carried out by the network of NODCs that supervise the national availability of research and environmental monitoring data provided, respectively, by research institutes and environmental agencies (Fig. 2). NODCs maintain regular contact with data originators collecting and enriching data with the best set of relevant metadata to ensure the reliability of the information. NODCs are also responsible for the first QC of data, flagged with quality information.

Data access is regulated by a data policy (defined in agreement with data originators) which aims to establish a balance between the right of the originator to get proper acknowledgment for data acquisition and the need for open access through free and unrestricted exchange of data, meta-data, and data products. The analysis of data policies for EMODnet Chemistry data shows differences between data access restrictions for nutrients and contaminants (Fig. 3).

Data requests from registered users are handled by NODCs through a data policy management system. Unrestricted data are freely available while restricted data need negotiation with data originators. This kind of filter on data access is an effective way to establish contacts and trust between data originators and data management centres, ensuring correct acknowledgement, which ultimately encourages data sharing.

By maximizing the availability of data to a larger community, SeaDataNet promotes the use of these data, thereby ensuring that their maximum value can be realized and thus contribute to increase knowledge of the marine environment. Figure 4 shows temporal distribution of nutrient data, spanning from 1900 to 2016; Table 1 shows the number of stations for parameters with more data available.

\section{Data quality}

The quality of the data is a key issue when merging heterogeneous data coming from different sources, periods, and geographic areas. Within EMODnet Chemistry community, commonly agreed and standardized data QC protocols have been defined (Holdsworth, 2010) to guarantee consistency among comprehensive databases which include data from different and/or unknown origin and covering long time periods. As a first step, the data are checked and completed by collators with a standard set of metadata that provide the basic information necessary for their long-term use. Afterwards, data undergo a validation loop which consists in several validation steps. The first is done by data collators, prior to the inclusion in the decentralized infrastructure, and the 


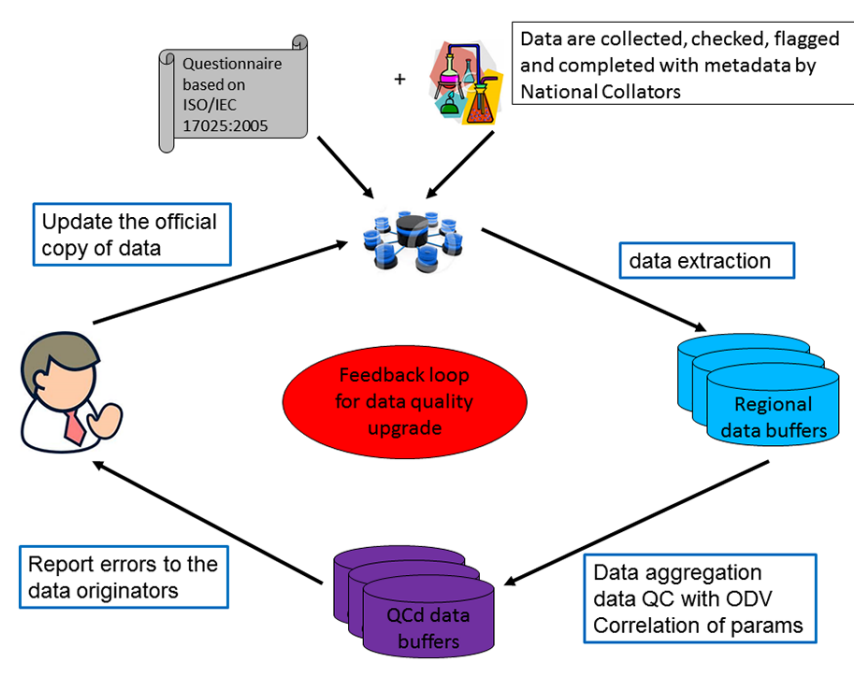

Figure 5. Data validation loop.

second step, which consists of regional quality control, is performed at regional scale on aggregated datasets. The first QCs ensure that position and time of data are realistic and compare measurements with broad ranges and specific regional ranges. Whenever available, data are also compared with climatology. As a result of the first QC step, all data are archived with a quality flag value that provides information about their reliability.

At this point, data aggregation and regional QC are performed at regional scale, following a common protocol. Data aggregation is done with the objective to unify the various analytic terms into a unique aggregated term with conversion to a unique measurement unit. The ODV software has a built-in aggregation procedure applying a number of business rules like possible units conversions. (Lowry et al., 2013)

The main goal of this activity is to obtain a harmonized dataset (e.g. a unique dataset of phosphate concentration in the water column starting from different datasets of phosphate concentration expressed with different units) that could be used to generate homogeneous data products. The results of the regional QC are sent to the data collators (NODCs) to correct errors or anomalies in the original copy of the data available in the EMODnet infrastructure. This feedback loop guarantees data quality upgrade (Fig. 5).

To improve and homogenize the QC procedures and standards adopted (at least at regional level), a QC survey has been carried out within EMODnet Chemistry community, in order to collect the best practices in data validation and highlight gaps of the different institutes involved (Vinci et al., 2015).

\section{Data products}

In order to accomplish the Marine Strategy Framework Directive requirements, EMODnet Chemistry developed prod- ucts suitable to visualize the time evolution of a selected group of measurements and to calculate spatially distributed data products specifically relevant for MSFD descriptor 5 (eutrophication), 8 (chemical pollution), and 9 (contaminants in seafood) as typically done with satellite data (Colella et al., 2016; Gohin et al., 2008)

The interpolated maps have been produced with the Variational Inverse Method (VIM; Brasseur et al., 1996), using the software DIVA (Data-Interpolating Variational Analysis; Troupin et al., 2010). DIVA is an appropriate numerical implementation of VIM suitable for oceanographic data spatial analysis as it is designed to obtain a gridded field from the availability of non-uniformly distributed observations (Barth et al., 2010; Troupin et al., 2012).

Interpolated maps are now generated, mainly for nutrients, with 10-year moving window in order to find a balance between the duration of the environmental evaluation cycle for member states (to provide maps with a time frame near to the 6-year process of the member states' evaluation) and the number of years that guarantee a sufficient data coverage.

An example of a visualization useful for the assessment of eutrophication and in particular of nutrient concentration in the water column is presented in Fig. 6, which displays surface distribution of phosphate concentration in spring for the decade 2003-2013 (centred in 2008).

Profiles and time series plots are automatically generated from the regional aggregated and validated datasets (called regional buffers), thanks to a service based on WPS OGC standard, and can be dynamically customized (Fig. 7).

There are ongoing efforts to develop a more efficient information management thanks to a system of data buffers hosted in a cloud system. Data are harvested and validated in buffers and are then used for product generation.

\section{Conclusions and perspectives}

EMODnet is a long-term marine data initiative developed through a stepwise approach aiming to ensure that European marine data will become easily accessible, interoperable, and free of restrictions on use. EMODnet Chemistry started in 2009 to fulfill EU Marine Strategy Framework Directive requirements for the assessment of eutrophication and contaminants, following EU INSPIRE Directive rules (European Commission, 2007).

With the start of EMODnet phase II, DG MARE and DG ENV started a coordination table to agree on a joint process and to identify how EMODnet can best contribute in practical terms to the MSFD. EMODnet Chemistry implemented a set of recommendations, in communication with RSCs contracting parties. The situation is not homogeneous in EU sea basins. While much of the chemistry and contaminant data are well organized within OSPAR Commission and Helsinki Convention (HELCOM), namely in the North Sea and Baltic Sea, respectively, EMODnet Chemistry has a more useful 


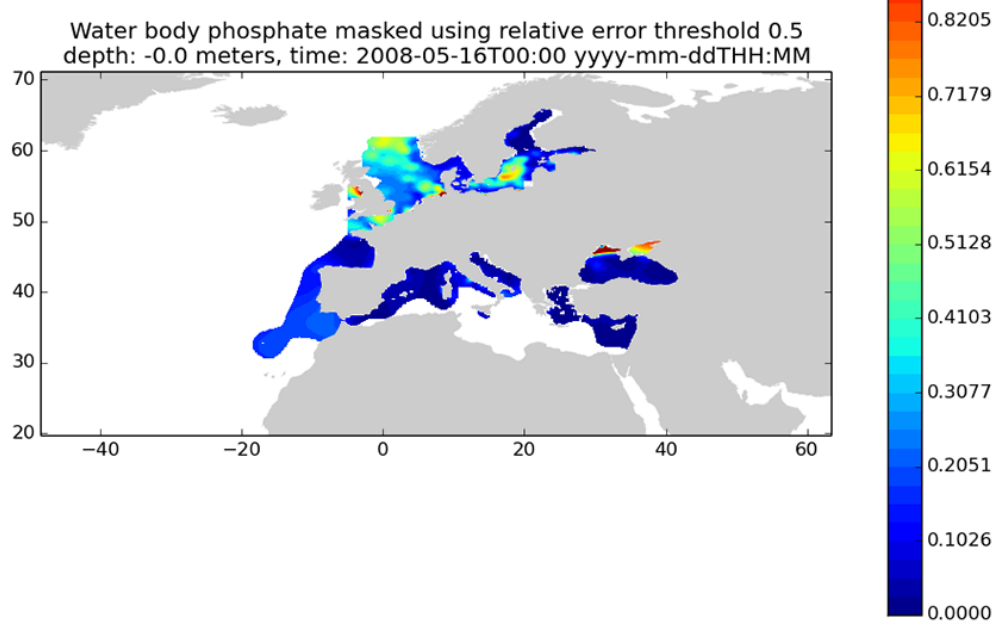

Figure 6. Ten-year average of water body phosphate concentration $\left(\mu \mathrm{mol} \mathrm{PL} \mathrm{PL}^{-1}\right)$ in the surface layer for all EU sea basins (years $\left.2003-2013\right)$. Interpolated maps can be selected from the Ocean Browser viewing service interface with the following steps: choose "Select data products" button, scroll and choose in the pop-up window from the list of available products, and then select "Add layer" in the lower left corner of the pop-up window. Maps can also be downloaded in different formats obtaining results as in this example (PNG file).

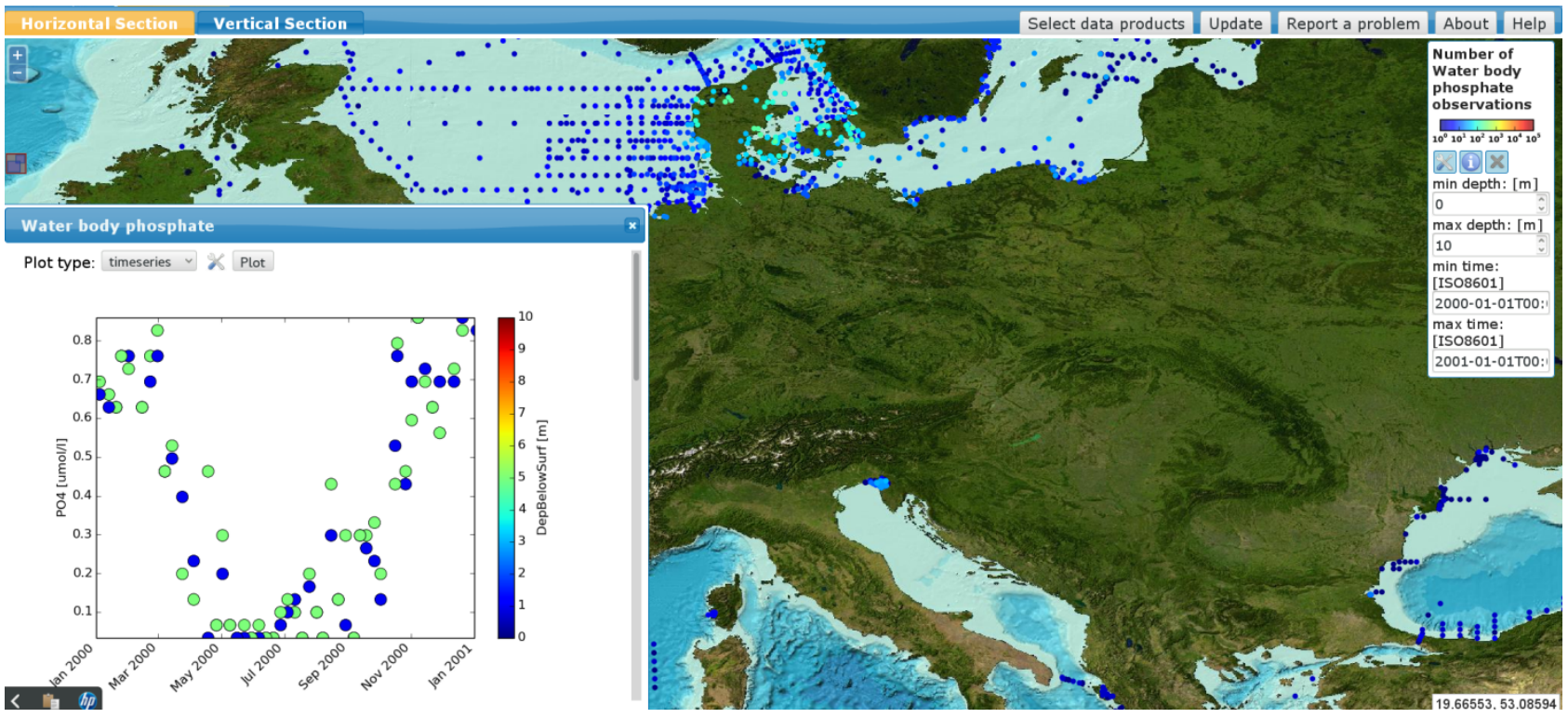

Figure 7. Screenshot from the web portal showing the time series dynamically plotted and visualized thanks to the OGC WPS services.

role in the Mediterranean where these outputs are less well organized. A Memorandum of Understanding with the Commission on the Protection of the Black Sea against Pollution (Bucarest Convention) is under preparation to formalize the cooperation in terms of providing dedicated access to EMODnet Chemistry regional products for supporting management of MSFD indicators as well as increasing participation in the advisory groups meetings. A similar step is under discussion with the Information and Communication
Regional Activity Center (INFO-RAC) through the United Nations Environmental Programme, Coordinating Unit for the Mediterranean Action Plan for the Barcelona Convention (UNEP/MAP).

These on-going efforts show the importance of EMODnet Chemistry results and the extensions that might be planned in view of the last EMODnet implementation phase aiming at a full resolution. 
In the next years, EMODnet Chemistry could play an important role in the European environmental reporting landscape with two main tasks. The first task consists in providing standardized and quality-checked buffers of data for specific Regions. The second task is to act as an umbrella providing standards, best practices, and infrastructure to aggregate at regional level the single member states.

\section{Data availability}

Data presented in the paper are available, according to specific data policies (defined in agreement with data originators), at http://www.emodnet-chemistry.eu/data_access.html. Aggregated data products (GIS layers) are public domain and freely available for all users. They are generated by EMODnet Chemistry under the support of DG MARE Call for Tenders 2008/03 and 2012/10.

Competing interests. The authors declare that they have no conflict of interest.

Acknowledgements. This work was supported by DG MARE, Call for Tenders 2008/03 and 2012/10. Authors acknowledge the contribution of the whole EMODnet Chemistry partnership, involved in the technical infrastructure development, in marine chemical data gathering, harmonization, standardization and quality control, and in data products preparation, as well as all data providers.

Edited by: I. Federico

Reviewed by: two anonymous referees

\section{References}

Barth, A., Alvera-Azcárate, A., Troupin, C., Ouberdous, M., and Beckers, J.-M.: A web interface for griding arbitrarily distributed in situ data based on Data-Interpolating Variational Analysis (DIVA), Adv. Geosci., 28, 29-37, doi:10.5194/adgeo-28-292010, 2010.

Brasseur, P., Beckers, J. M., Brankart, J. M., and Schoenauen, R.: Seasonal temperature and salinity fields in the Mediterranean Sea: Climatological analyses of a historical data set, Deep-Sea Res. Pt. I, 43, 159-192, doi:10.1016/0967-0637(96)00012-X, 1996.

Colella, S., Falcini, F., Rinaldi, E., Sammartino, M., and Santoleri, R.: Mediterranean Ocean Colour Chlorophyll Trends, PLoS ONE, 11, e0155756, doi:10.1371/journal.pone.0155756, 2016.

Dupont, C., Belin, A., Moreira, G., Vermonden, B., Cochrane, S., Wilson, L., Emblow, C., Kater, B., Des Clercs, S., Parr, W., Le Visage, C , Green, N., Cools, J., and Thomsen, F.: Article 12 Technical Assessment of the MSFD 2012 obligations Mediterranean Sea, Contract No. 070307/2012/634823/SER/D2 - Task F, 41 pp., 2014.
European Commission: Green Paper - Towards a future Maritime Policy for the Union: A European vision for the oceans and seas, COM (2006) 275 final, Volume II - ANNEX, 49 pp., 2006.

European Commission: Directive 2007/2/EC of the European Parliament and of the Council of 14 March 2007 establishing an Infrastructure for Spatial Information in the European Community (INSPIRE), 14 pp., 2007.

European Commission: Directive 2008/56/EC of the European Parliament and of the Council of 17 June 2008, establishing a framework for community action in the field of marine environmental policy (Marine Strategy Framework Directive), 22 pp., 2008.

European Commission: Green Paper - Marine Knowledge 2020 - 45 from seabed mapping to ocean forecasting, COM (2012) 473 final, doi:10.2771/4154, 23 pp., 2012.

Gohin, F., Saulquin, B., Oger-Jeanneret, H., Lozac'h, L., Lampert, L., Lefebvre, A., and Bruchon, F.: Towards a better assessment of the ecological status of coastal waters using satellitederived chlorophyll- $a$ concentrations, Remote Sens. Environ., 112, 3329-3340, 2008.

Holdsworth, N.: EMODnet Chemistry QC and QA basic guidelines, 11 pp., doi:10.6092/3cb41f4c-c401-45a7-ab32-0abd373e03bb, 2010.

Lowry, R., Leadbetter, A., and Vinci, M.: Semantically-enhanced Aggregation of SeaDataNet Data, Boll. Geof. Teor. Appl., Vol. 54 Supp. (2013), IMDIS 2013 International Conference on Marine Data and Information Systems, 23-25 September 2013, Lucca (Italy), Book of Abstracts, 49-51, 2013.

Palialexis, A., Tornero, V., Barbone, E., Gonzalez, D., Hanke, G., Cardoso, A. C., Hoepffner, N., Katsanevakis, S., Somma, F., and Zampoukas, N.: In-Depth Assessment of the EU Member States' Submissions for the Marine Strategy Framework Directive under articles 8, 9 and 10, Report EUR 26473 EN EU, JRC-IES, 149 pp., doi:10.2788/64014, 2014.

Troupin, C., Machí n, F., Ouberdous, M., Sirjacobs, D., Barth, A., and Beckers, J. M.: High-resolution Climatology of the North-East Atlantic using Data-Interpolating Variational Analysis (Diva), J. Geophys. Res., 115, C08005, doi:10.1029/2009JC005512, 2010.

Troupin, C., Barth, A., Sirjacobs, D., Ouberdous, M., Brankart, J. M., Brasseur, P., Rixen, M., Alvera-Azcárate, A., Belounis, M., Capet, A., Lenartz, F., Toussaint, M. E., and Beckers, J. M.: Generation of analysis and consistent error fields using the Data Interpolating Variational Analysis (DIVA), Ocean Model., 52-53, 90-101, 2012.

Vinci, M., Giorgetti, A., and Brosich, A.: New EU efforts to assess the state of the marine environment: the Emodnet Chemistry pilot project, Boll. Geof. Teor. Appl., Vol. 54 Supp. (2013), IMDIS 2013 International Conference on Marine Data and Information Systems, 23-25 September 2013, Lucca (Italy), Book of Abstracts, 121-122, 2013.

Vinci, M., Giorgetti, A., and Lipizer, M.: EMODnet Chemistry 2 Quality Control inventory, 74 pp., doi:10.6092/97a6685c-99f34986-8285-f711e4101a99, 2015. 